LA-UR-01-6766

Approved for public release; distribution is unlimited.

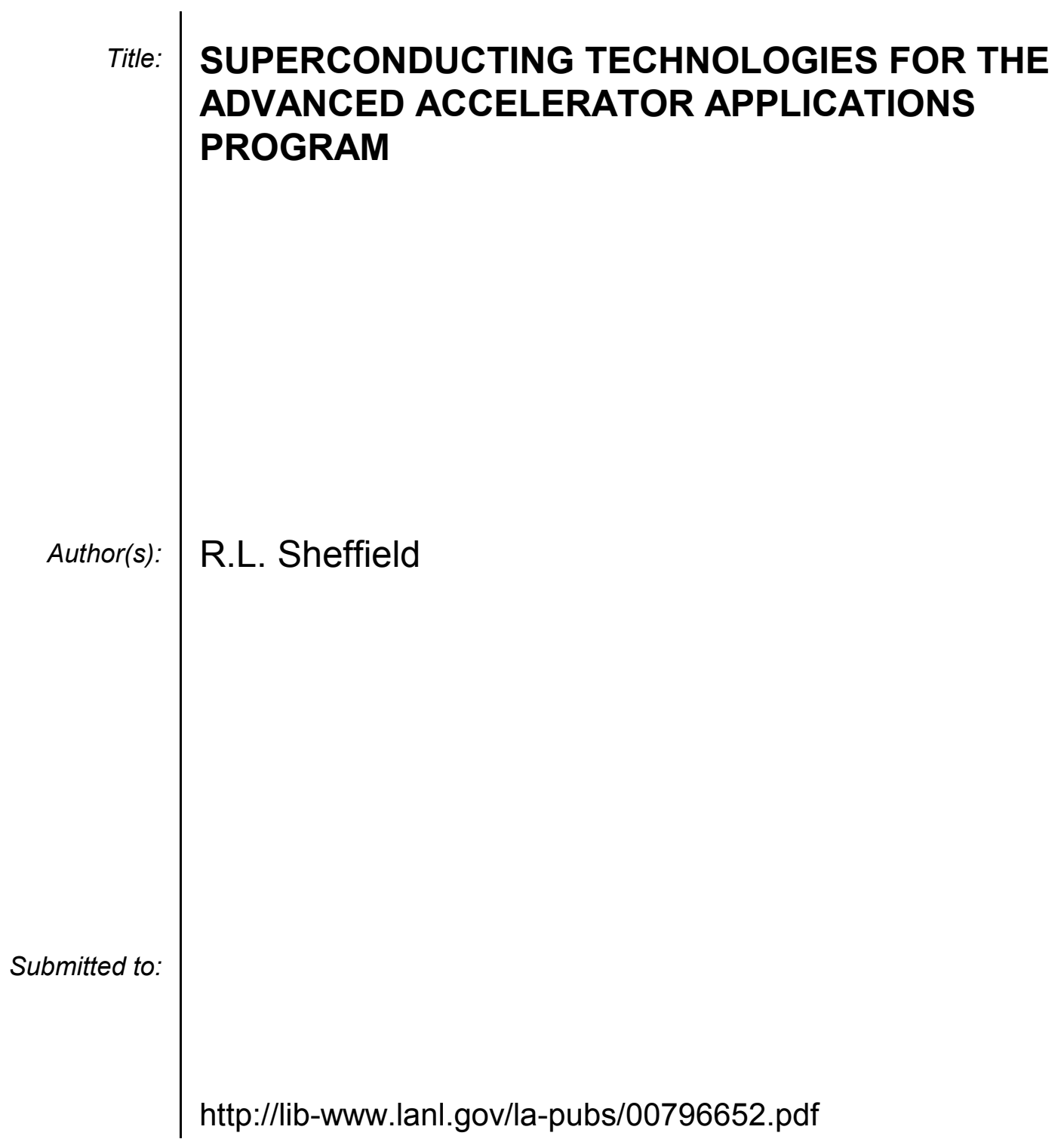

Los Alamos National Laboratory, an affirmative action/equal opportunity employer, is operated by the University of California for the U.S. Department of Energy under contract W-7405-ENG-36. By acceptance of this article, the publisher recognizes that the U.S. Government retains a nonexclusive, royaltyfree license to publish or reproduce the published form of this contribution, or to allow others to do so, for U.S. Government purposes. Los Alamos National Laboratory requests that the publisher identify this article as work performed under the auspices of the U.S. Department of Energy. Los Alamos National Laboratory strongly supports academic freedom and a researcher's right to publish; as an institution, however, the Laboratory does not endorse the viewpoint of a publication or guarantee its technical correctness. 


\title{
SUPERCONDUCTING TECHNOLOGIES FOR THE ADVANCED ACCELERATOR APPLICATIONS PROGRAM*
}

\author{
R.L. Sheffield, LANL, Los Alamos, NM 87545, USA
}

\section{Abstract}

The Advanced Accelerator Applications Program goal is develop technologies required for transmuting radioactive waste into shorter lived, less-toxic material. As part of this program, an accelerator provides the required hard neutron spectrum through a spallation process. The proton beam must have a high-reliability at continuous currents levels up to $13-\mathrm{mA}$. The accelerator front end is based on LEDA, a $75-\mathrm{KeV}$ injector followed by a $6.7-\mathrm{MeV}$ RFQ. Because of the low-current and high-reliability requirement, the remaining accelerator structure is superconducting. From $6.7-\mathrm{MeV}$ to $109-\mathrm{MeV}$, the accelerator uses spoke resonators with betas of 0.175 and 0.34 . From $109-\mathrm{MeV}$ to the final energy of $600-\mathrm{MeV}$, the accelerator uses elliptical cavities with betas of 0.48 and 0.64 .

\section{AAA PROGRAM OVERVIEW [1]}

Authorized by United States (US) Congress in fiscal year 2001, the Advanced Accelerator Application (AAA) program was created to address pressing nuclear issues facing the US. These issues include nuclear energy and waste management concerns, declining US nuclear infrastructure, and global nuclear leadership.

A key roadblock to development of additional nuclear power capacity is a concern over management of nuclear waste. Nuclear waste is predominantly comprised of used fuel discharged from operating nuclear reactors. The roughly 100 operating US reactors, that currently produce about $20 \%$ of the US electricity, will create about 87,000 tons of such discharged or "spent" fuel over the course of their lifetimes. Sixty thousand tons of this waste is destined for geologic disposal at the Yucca Mountain site in Nevada. Worldwide, more than 250,000 tons of spent fuel from reactors currently operating will require disposal. The toxicity of the spent fuel, mainly due to ionization radiation, will affect future generations for millions of years. The large quantity and its long-lived toxicity present great difficulties to waste management.

Nearly all issues related to risks to future generations arising from long-term disposal of such spent nuclear fuel is attributable to approximately $1 \%$ of its content. This $1 \%$ is made up primarily of transuranic elements and longlived fission products. Transuranic elements are typically plutonium, neptunium, americium, and curium produced

\footnotetext{
* Work supported by the US Department of Energy
}

by neutron captures in uranium fuel. Long-lived fission products are typically iodine and technetium created as products from fission processes. If we can reduce or eliminate this $1 \%$ of the spent fuel, the toxic nature of the spent fuel drops below that of the natural uranium ore that was originally mined for nuclear fuel.

This $1 \%$ of the spent fuel can be disposed of using a combination of two approaches. First, fertile elements like plutonium can be fabricated into nuclear fuel to produce energy in a reactor. Second, the rest can be most efficiently eliminated through nuclear transmutation using fast neutrons produced by a spallation process initiated with a proton beam produced by an accelerator. The goal of AAA program is to develop technologies required for transmuting radioactive waste using spallation neutrons into shorter-lived, less-toxic material. Such technology development can be carried out in a facility, the Accelerator Demonstration Test Facility (ADTF), as envisioned in Figure 1. The primary components of ADTF are a proton linac, a spallation neutron source, and a subcritical multiplier where transmutation of nuclear waste occurs.

\section{AAA LINAC REQUIREMENTS [2]}

AAA program requires a hard neutron spectrum that can be produced by a spallation process from a proton beam. The proton beam for the ADTF linac must have highreliability at continuous-current levels up to $13 \mathrm{~mA}$.

The power of the ADTF linac is determined by the design of the subcritical multiplier. With a reactor equivalent power of $100 \mathrm{MW}$ and with the multiplier $\mathrm{k}_{\mathrm{eff}}$ in a range of 0.92 to 0.98 , the maximum proton beam power is $8 \mathrm{MW}$.

The beam energy of the linac is determined by the profile of power density deposition of the proton beam in a spallation target. A uniform profile is needed to reduce excessive local heating. Figure 2 shows the profiles of deposited power density in tungsten for different proton beam energies. The power density profile is characterized by a smooth curve ending with the Bragg peak where the proton stops in the tungsten. At low proton beam energies, the peak power density is defined by the maximum of the Bragg peak. At high beam energies, $\mathrm{dE} / \mathrm{dx}$ of nuclear processes leads to power peaking within a few centimeters of the front face of the tungsten target. A beam energy of 
$600 \mathrm{MeV}$ is chosen for the ADTF linac because the power density is roughly the same at the target front and at the location of the Bragg peak.

The beam current of the ADTF linac is determined by the neutron yield. The number of spallation neutrons produced per proton is shown in Figure 3 as a function of proton beam energy. Beyond $500 \mathrm{MeV}$, neutron yield per proton increases linearly with proton beam energy. This linear dependence, extrapolated towards low proton beam energy, has a $240-\mathrm{MeV}$ offset because of nuclear reaction thresholds of neutron production. Simulations showed that spallation from heavy metals $(\mathrm{Ta}, \mathrm{W}, \mathrm{Hg}, \mathrm{Pb}, \mathrm{Bi}$ ) produce about the same number of neutrons. Neutron production from uranium is about $60 \%$ greater than heavy metals due to fission cross sections at neutron energies below $1 \mathrm{MeV}$. Using neutron yields in Figure 3 and a $k_{\text {eff }}$ of 0.92 , a reactor equivalent power of $100 \mathrm{MW}$ can be produced using a beam current $13 \mathrm{~mA}$.



Figure 1 A schematic layout of the Accelerator Demonstration Test Facility.

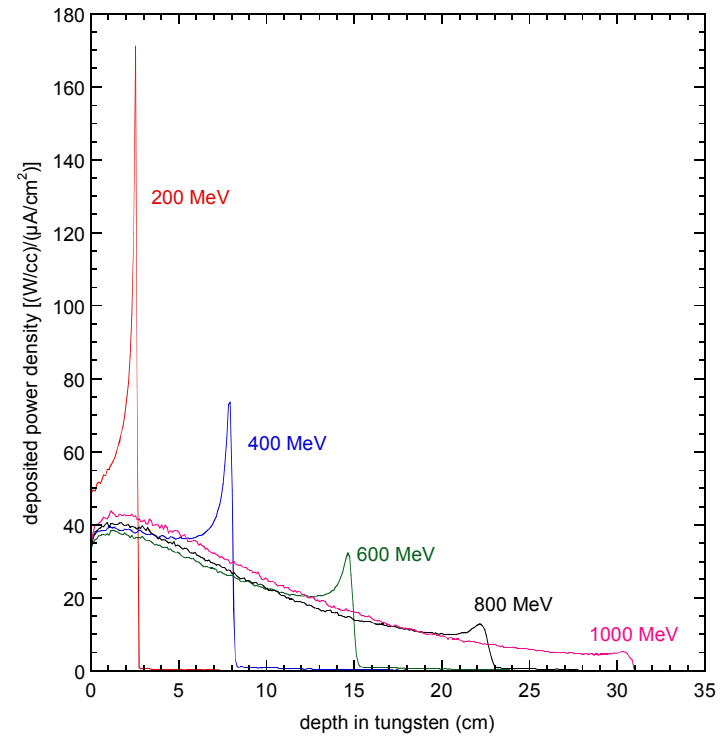

Figure 2 Profiles of deposited power density as a function of depth in a tungsten spallation target for proton beam of various energies.

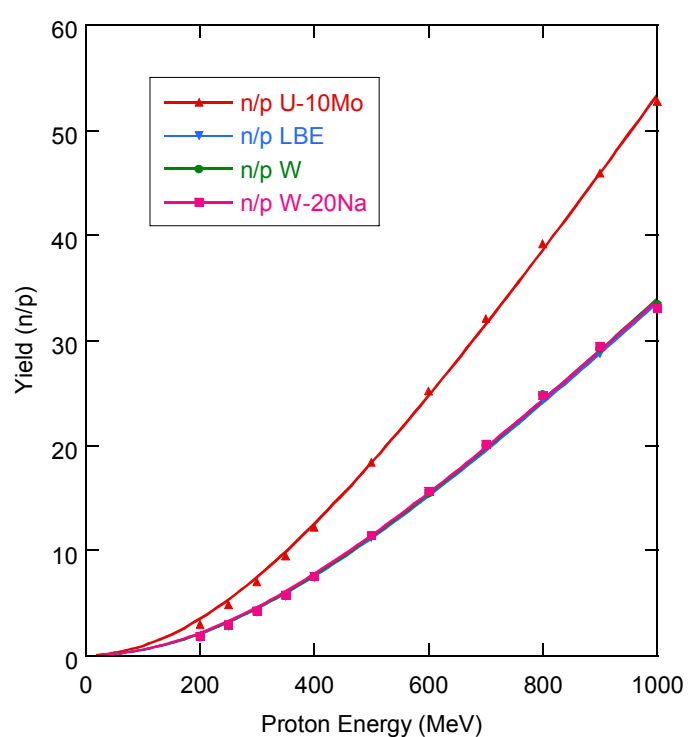

Figure 3 Number of spallation neutrons per proton as a function of proton beam energy. 
For additional linac requirements, ADTF linac needs to consider the following. First, designers have identified a subcritical multiplier intolerance to beam interrupts longer than $300 \mathrm{~ms}$. Second, advances in super-conducting radiofrequency (SCRF) technology combined with the low beam current of $13 \mathrm{~mA}$ makes the application of SCRF linac technology very cost effective. Third, the engineering challenges lie in the safe, controlled coupling of an accelerator to a subcritical reactor through a spallation target. System control and safe operation will demand the understanding and resolution of the potentially complex behavior of this coupled accelerator/target/reactor system.

\section{AAA LINAC DESIGN}

A SCRF linac has been chosen for the ADTF linac because, compared to linacs using traditional roomtemperature (RT) copper technology, SCRF linacs are more power efficient and expected to have higher reliability. A comparison of SCRF and RT technologies has been reviewed recently at Los Alamos by a panel of accelerator experts [3]. A superconducting linac significantly lowers the total linac $\mathrm{AC}$ power requirement. For a $600-\mathrm{MeV}$ linac at $13 \mathrm{~mA}$, a SCRF linac needs 23 $\mathrm{MW}$ of $\mathrm{AC}$ power as compared to $80 \mathrm{MW}$ needed for a RT linac. The SCRF linac will employ independently controlled RF modules with redundancy, allowing the less than 300 ms adjustment of RF phases and amplitudes of $\mathrm{RF}$ modules to compensate for faults of individual cavities, klystrons, or focusing magnets. The SCRF cavities will have larger bore radius that relaxes alignment and steering tolerances, as well as reducing beam loss. Thermal transient has been a major cause of out-of-lock trips in RT linacs. Operating at a stable cryogenic temperature, SCRF linacs are expected to have a reduced number of such trips.

Table 1 shows the ADTF linac architecture. Except for the room-temperature RFQ, the linac is made up of superconducting sections of spoke cavities and elliptical cavities designed to match the proton energy (also referred to by $\beta$, the proton velocity divided by the speed of light) at the section location. Except for the spoke cavities, the linac technology used has all been demonstrated. The injector and the RFQ have been fabricated and tested in the Los Alamos Low-Energy Demonstration Accelerator (LEDA) [4]. The elliptical cavities, both the medium-energy cavities $[5,6]$ and the power couplers [7.8], have shown excellent performance in $R \& D$ for the Accelerator Production of Tritium project. A prototype cryomodule has also been fabricated.

Table 1 Linac architecture of ADTF linac

\begin{tabular}{|c|c|c|c|c|c|}
\hline RFQ & Spoke & Spoke & Spoke & Elliptical & Elliptical \\
\hline $350 \mathrm{MHz}$ & $350 \mathrm{MHz}$ & $350 \mathrm{MHz}$ & $350 \mathrm{MHz}$ & $700 \mathrm{MHz}$ & $700 \mathrm{MHz}$ \\
\hline & 2 -gap & 3 -gap & 3 -gap & 5 -cell & 5 -cell \\
\hline & $\begin{array}{c}\beta_{\mathrm{g}}=0.17 \\
5\end{array}$ & $\beta_{\mathrm{g}}=0.20$ & $\beta_{\mathrm{g}}=0.34$ & $\beta_{\mathrm{g}}=0.50$ & $\beta_{\mathrm{g}}=0.64$ \\
\hline $6.4 \mathrm{MeV}$ & $14 \mathrm{MeV}$ & $40 \mathrm{MeV}$ & $104 \mathrm{MeV}$ & $211 \mathrm{MeV}$ & $600 \mathrm{MeV}$ \\
\hline
\end{tabular}

Spoke cavities are low-energy SCRF structures developed originally at Argonne national Laboratory. It has been recently adopted for the RIA (rare-isotope accelerator) linac. Development of spoke cavities in Los Alamos [9] was started in spring of 2001. Excellent results were obtained for single cell spoke cavities [10]. Figure 4 shows an engineering model of a multi-cell spoke cavity.

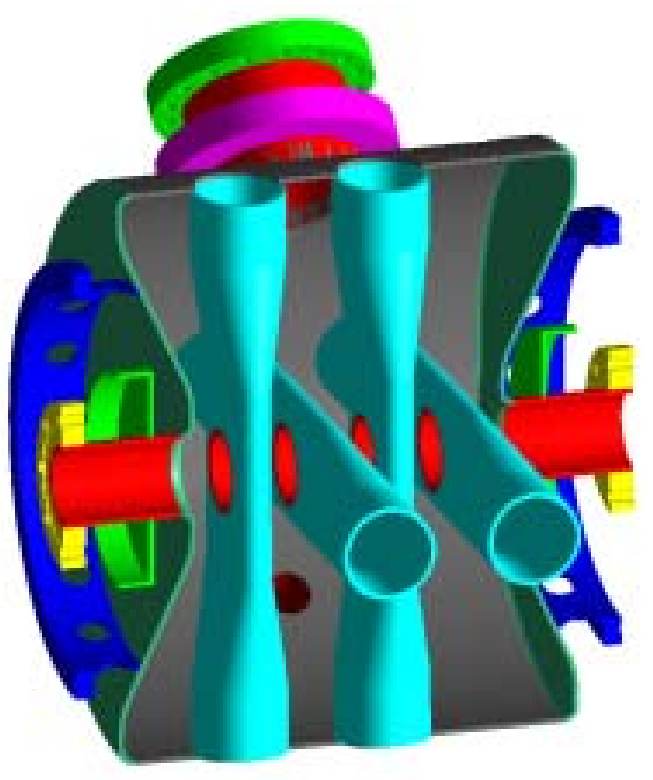

Figure 4 Engineering model of a $\beta=0.125$, 5-gap spoke cavity at $350 \mathrm{MHz}$

An ADTF SCRF linac has higher availability and reliability because of the short cavities and RF-system design allows for quick recovery from a RF-system failure, which is a major cause of linac unreliability. The RF system for the ADTF linac is designed to operate with failed cavities. The RF windows and all transmission-line components can be isolated if a failure is anticipated by observing an increased arc rate or increase in temperature of the RF window. If such incident occurs, the associated cavities will be detuned. Cavities downstream will be adjusted in phase and amplitude to make up for detuned cavities. This strategy will minimize the interruption of the beam by RF-system failures to less than 300 milliseconds as required. While the linac operation continues, the failed RF station will be repaired, tested, and made ready for return to service.

\section{SUMMARY}

The large nuclear waste-disposal challenge is driven by a relatively small amount of long-lived materials. Their removal makes long-term disposal of remaining less toxic materials straightforward and robust. The lifetime of the removed highly-toxic long-lived materials can then be reduced from millions of years to hundredths of years by transmutation technology based on accelerator produced 
spallation neutrons. Transmutation programs such as AAA offer a path to significantly reducing the volume and halflife of nuclear waste destined for geologic storage. SCRF linacs, with high availability and reliability is a suitable choice for a $600 \mathrm{MeV}, 13 \mathrm{~mA}$ proton linac as required for the AAA program

\section{REFERENCES}

[1] http://aaa.lanl.gov

[2] E. Pitcher, private communication.

[3] "Accelerator-Driven Test facility Linac Review; April 10-12, 2001," Los Alamos National Laboratory report LA-UR-01-2834, May 2001.

[4] H. V. Smith Jr. and J. D. Schneider, "Status report on the Low-Energy Demonstration Accelerator (LEDA)," LINAC 2000, Monterey, CA, USA, August 21-25.
[5] T. Tajima, et al., "Test Results of $\beta=0.64,700 \mathrm{MHz}$, 5-cell elliptical Cavities," this Workshop.

[6] "Executive Summary; Development and performance of Medium-b Superconducting cavities," Los Alamos National Laboratory report LA-CP-01-0202, April 2001

[7] E. N. Schmierer, et al, "Results of the APT RF Power Coupler Development for Superconducting Linacs, this Workshop.

[8] "Executive Summary; Development and Performance of Superconducting-Cavity Power couplers," Los Alamos National Laboratory report LA-CP-01-461, August 2001.

[9] F. L. Krawczyk, "Design of a $\beta=0.175$ 2-Gap spoke Resonator," this Workshop

[10] T. Tajima, "Status of the LANL Activities in the Filed of RF Superconductivity," this Workshop. 